\title{
Co-treatment of LY294002 or MK-2206 with AZD5363 Attenuates AZD5363-induced Increase in the Level of Phosphorylated AKT
}

\author{
AE-RAN CHOI ${ }^{1 *}$, JU-HWA KIM ${ }^{1 *}$, YEON HWA WOO ${ }^{1}$, JI HYUN CHEON ${ }^{2}$, \\ HYUNG SIK KIM ${ }^{2}$ and SUNGPIL YOON ${ }^{1,2}$ \\ ${ }^{1}$ Research Institute, National Cancer Center, Goyang-si, Republic of Korea; \\ ${ }^{2}$ School of Pharmacy, Sungkyunkwan University, Suwon, Republic of Korea
}

\begin{abstract}
Clinical trials are in progress on AZD5363, an inhibitor of protein kinase $B(A K T)$, to assess its effects on the phosphoinositide 3-kinase (PI3K)/AKT/mammalian target of rapamycin (mTOR) pathway. Cells treated with AKT inhibitors have been reported to activate alternative pathways in order to escape growth inhibition. AZD5363-sensitized Hs578T breast cancer cells displayed reduced levels of phosphorylated glycogen synthase kinase 3 beta ( $p G S K 3 \beta)$. Interestingly, in AZD5363-treated cells, the level of phosphorylated (activated) $A K T(p A K T)$ increased. Since $p A K T$ positively correlates with cancer growth and survival, we aimed to identify conditions that could reduce AZD5363-induction of pAKT. We examined whether AZD5363 induction of pAKT could be reduced by cotreatment with inhibitors of the PIBK/AKT/mTOR pathway (LY294002, MK-2206, wortmannin, perifosine, rapamycin, everolimus, and temsirolimus). We observed that co-treatment of LY294002 or MK-2206 with AZD5363 reduced the level of pAKT. Since MK-2206 is clinically used, we propose that cotreatment using MK-2206 with AZD5363 would prove beneficial in blocking the AZD5363-induced pAKT signaling pathway. Our findings contribute to the development of AZD5363-based sensitization therapies for patients with cancer.
\end{abstract}

This article is freely accessible online.

*These Authors contributed equally to this study.

Correspondence to: Hyung Sik Kim, Ph.D. and Sungpil Yoon, $\mathrm{Ph} . D$. School of Pharmacy, Sungkyunkwan University, 2066 Seoburo, Jangan-gu, Suwon, Gyeonggi-do, 16419, Republic of Korea. Tel: +82 312907789, Fax: +82 312928800, e-mail: hkims@skku.edu, syoon88@gmail.com.

Key Words: AZD5363, LY294002, MK-2206, pAkt, PI3K/AKT/ mTOR.
AZD5363 is an oral small-molecule, new-generation drug that potentially inhibits all three isoforms of the protein kinase $B$ (AKT) proteins: AKT1, AKT2, and AKT3 (1-4). Clinical trials are in progress to evaluate the influence of AZD5353 on PI3K/AKT/mTOR pathway. AZD5363 has been tested as an anticancer agent in various tumor models, such as breast cancer, prostate cancer, and leukemia (5-8). In addition, a recent clinical trial investigated the use of AZD5363 in patients with advanced solid tumors. Various synergistic studies were also performed for improving the efficacy of AZD5363 (5-16). Administration of AZD5363 in combination with inhibitors of autophagy, phosphoinositide 3-kinase (PI3K), anti-androgen drugs, and drugs such as trastuzumab, enzalutamide and gefitinib, was reported to increase cancer cell sensitization. Identification of the conditions that govern the mechanism of action of AZD5363 would be an important step in facilitating its therapeutic use in patients with cancer.

In the present study, we tested whether co-treatment of various inhibitors involved in the $\mathrm{PI} 3 \mathrm{~K} / \mathrm{AKT} / \mathrm{mammalian}$ target of rapamycin (mTOR) pathway would reduce AZD5363 induction of AKT phosphorylation (pAKT). We also investigated which of the PI3K/AKT/mTOR inhibitors better attenuated pAKT induction by AZD5363. Furthermore, we tested whether co-treatment with inhibitors affected other signaling pathways or increased apoptosis, in comparison to AZD5363 treatment alone. Our results contribute to increased efficiency of AZD5363 drug-based therapy for cancer.

\section{Materials and Methods}

Reagents. AZD5363, MK-2206, wortmannin, perifosine, rapamycin, everolimous, and temsirolimous were supplied by Selleckchem (Houston, TX, USA). LY294002 was supplied by Calbiochem (Bellerica, MA, USA). Vinblastine was purchased from Enzo Life Sciences (Farmimgdale, NY, USA).

Antibodies. Antibodies against AKT, pAKT, phosphorylated glycogen synthase kinase 3 beta (pGSK3 $\beta$ ), phosphorylated 
ribosomal protein S6 kinase beta-1 (p70S6K), phosphorylated pyruvate dehydrogenase lipoamide kinase isozyme 1 (pPDK1), phosphorylated eukaryotic translation initiation factor 4E-binding protein 1 (p4EBP1), proliferating cell nuclear antigen (PCNA), and cleaved poly ADP ribose polymerase (C-PARP) were from Cell Signaling Technology (Danvers, MA, USA). Antibodies against glyceraldehyde 3-phosphate dehydrogenase (GAPDH), survivin, and phosphorylated retinoblastoma protein $(\mathrm{pRb})$ were from Santa Cruz Biotechnology (Santa Cruz, CA, USA). Antibodies against pmTOR were from Abcam (Cambridge, UK). Antibody against cyclin D1 was from Biosource (Camarillo, CA, USA).

Cell culture. Hs578T breast cancer cells were obtained from the Korean Cell Line Bank (Seoul, South Korea), and were used previously (17-19). Human oral squamous carcinoma cell line, KB and its multidrug-resistant subline, KBV20C, were obtained from Dr. Yong Kee Kim, and were previously described (20). All cell lines were cultured in RPMI-1640 containing 10\% fetal bovine serum, $100 \mathrm{U} / \mathrm{ml}$ penicillin, and $100 \mu \mathrm{g} / \mathrm{ml}$ streptomycin (WelGENE, Daegu, South Korea). Cells were grown on 6-well plates for 1 day, and then treated with drugs or $0.1 \%$ dimethylsulfoxide (control).

Western blot analysis. Total cellular proteins were extracted using a previously described trichloroacetic acid (TCA) method $(17,18)$. Briefly, cells grown in $60-\mathrm{mm}$ dishes were washed three times with $5 \mathrm{ml}$ phosphate-buffered saline (PBS). Next, $500 \mu \mathrm{l}$ of $20 \%$ TCA were added to each plate. The cells were then dislodged by scraping and transferred to Eppendorf tubes. Proteins were pelleted by centrifugation for $5 \mathrm{~min}$ at $600 \times g$ and resuspended in $1 \mathrm{M}$ Tris- $\mathrm{HCl}$ $(\mathrm{pH}$ 8.0) buffer. The total protein concentrations were estimated using the dye-binding method. The proteins were resolved by sodium dodecyl sulfate-polyacrylamide gel electrophoresis (SDS-PAGE) and subjected to western blot analysis as previously described $(17,18)$.

Microscopic observation. Cells were grown on 6-well plates for 1 day, and then treated with drugs or $0.1 \%$ dimethylsulfoxide (control) for 1 or 2 days. The medium was removed, and PBS was added to each dish. Cells were examined immediately using an Axio observer.Z1 fluorescence inverted microscope (Carl Zeiss, Oberkochen, Germany) with a $5 \times$ or $10 \times$ objective lens (Carl Zeiss EC Plan-Neofluar). We performed two independent experiments.

\section{Results}

AZD5363-treated cancer cells display reduced level of $p G S K 3 \beta$, but increased level of pAKT. AKT-targeting drugs specifically sensitize cancer cells since they depend on AKT signaling for growth or survival. Firstly, in order to identify an AZD5363-susceptible cancer cell line, we tested growth inhibition of three cancer cell lines, KB (vinblastine-sensitive cell line), KBV20C (vinblastine-resistant cell line), and Hs578T cells. As shown in Figure 1A and 2A, Hs578T cells were sensitive to inhibition by AZD5363, whereas both drugsensitive KB and drug-resistant KBV20C cell lines were not at similar concentrations. Furthermore, we analyzed mechanism of action of AZD5363 in the Hs578T breast cancer cell line.
Since AZD5363 targets the AKT signaling pathway, we evaluated whether AZD536 down-regulated expression of AKT pathway-related proteins. As shown in Figure 1B, AZD5363 reduced levels of AKT, pGSK3 $\beta$, and pRb protein. Interestingly, the level of pAKT markedly increased in response to AZD5363 treatment. This suggests that the phosphorylation state of AKT had no effect on growth inhibition by AZD5363. It seems that AZD5363 affects AKT signaling pathway increasing the pAKT level, thereby inducing resistance to drug. Since an increased pAKT level could increase cellular growth signaling and survival to produce undesirable drug-resistant cell phenotypes, we investigated conditions that would attenuate AZD5363 induction of pAKT.

Co-treatment with LY294002 reduced AZD5363 induction of pAKT. We tested whether co-treatment with PI3K/AKT/ mTOR inhibitors attenuated pAKT induction by AZD5363. LY294002 is an established PI3K/AKT pathway inhibitor $(17,18)$. We observed that LY294002 inhibited the growth of and reduced the pGSK3 $\beta$ level in Hs578T cells, similarly to that induced by AZD5363 treatment (Figure 1C and Figure 2B). This suggests that PI3K/AKT pathway-targeting inhibitor could sensitize Hs578T cells.

Next, we tested whether AZD5363 induction of pAKT was attenuated by co-treatment with LY294002. Co-treatment of LY294002 with a high concentration of AZD5363 reduced the pAKT level (Figure 1C), indicating that PI3K/AKT inhibitor ameliorates the effect of AZD5363 on pAKT. This also suggests that co-treatment of LY294002 could reduce AZD5363-induced cellular growth or survival signaling. We analyzed this effect in detail. Two different concentrations of LY294002 and relatively lower concentrations of AZD5363 were used for an increased incubation period (up to 2 days) to determine whether prolonged co-treatment with LY294002 and AZD5363 reduced the pAKT level. As displayed in Figure 1D, AZD5363-induced increase in pAKT levels even at a lower concentration $(10 \mu \mathrm{M})$ and this increase was effectively reduced by both concentrations of LY294002 (10 $\mu \mathrm{M}$ and $20 \mu \mathrm{M})$. AZD5363 induction of pAKT was reduced by LY294002 in a dose-dependent manner (Figure 1D). Collectively, our results demonstrate that co-treatment of LY294002 reduces AZD5363 induction of pAKT.

Co-treatment with MK-2206 reduced AZD5363 induction of $p A K T$. We tested whether co-treatment with other AKT pathway inhibitors would reduce the AZD5363 induction of pAKT. For beneficial application purposes, we focused on clinically relevant inhibitors for co-treatment with AZD5363. Three known inhibitors MK-2206, wortmannin, and perifosine $(17,18,21-24)$, were selected for co-treatment. We observed that the effect of MK-2206, wortmannin, and perifosine were similar to that of AZD5363, and LY294002, 
$\mathbf{A}$

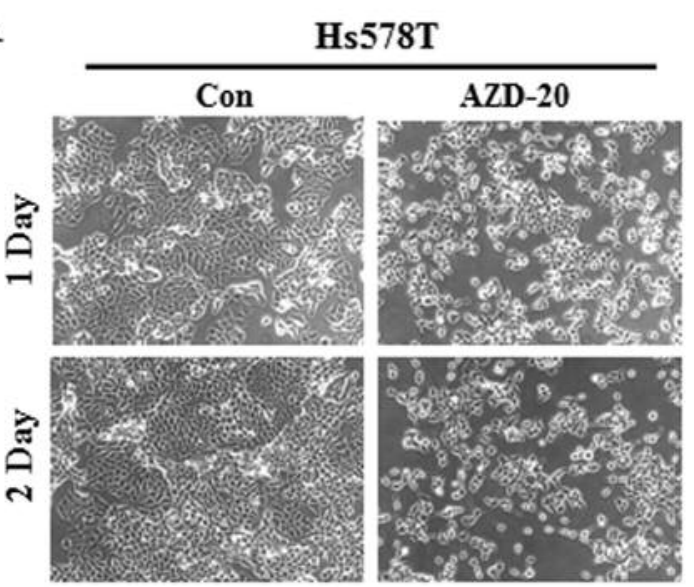

C

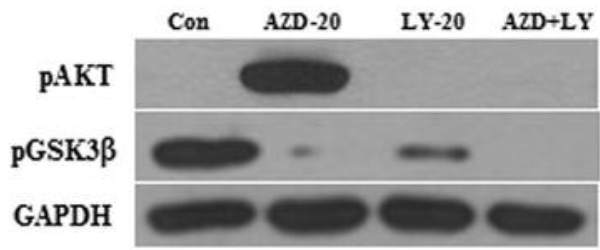

B

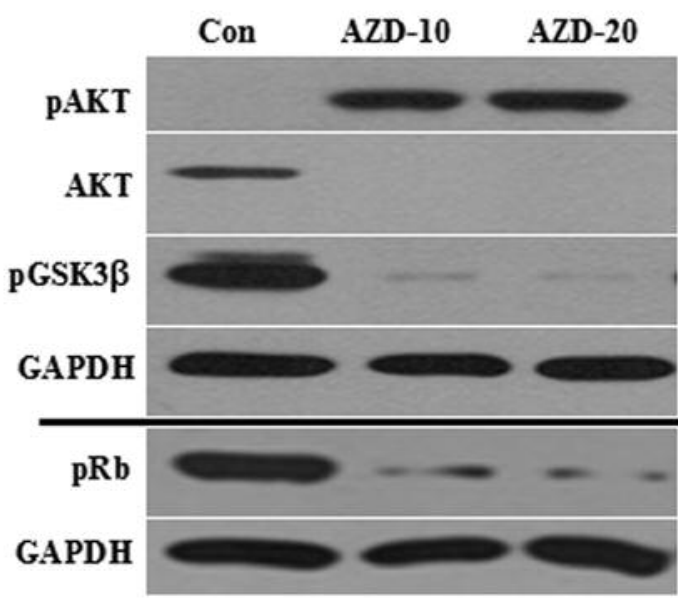

D
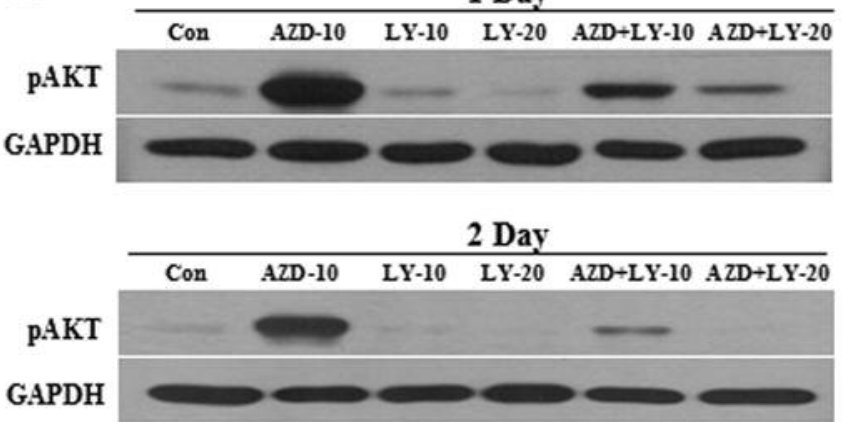

Figure 1. AZD5363 induction of phosphorylated protein kinase B (pAKT) in Hs578T breast cancer cells is reduced by LY294002. A: Hs578T cells were grown on 6-well plates and treated with $20 \mu \mathrm{M}$ AZD5363 (AZD-20) or 0.1\% dimethylsulfoxide (Con). After 1 or 2 days, all cells were observed using an inverted microscope with a $10 \times$ objective lens. B: Hs578T cell extracts were collected at 24 h after treatment with $10 \mu M$ or $20 \mu M$ AZD5363 (AZD-10, AZD-20), or 0.1\% dimethylsulfoxide (Con). They were then used for western blot analyses using antibodies against pAKT, AKT, phosphorylated glycogen synthase kinase 3 beta ( $p G S K 3 \beta)$, phosphorylated retinoblastoma protein ( $p$ Rb), and glyceraldehyde 3-phosphate dehydrogenase (GAPDH). C: Hs578T cell extracts were collected at 24 h after treatment with AZD-20, and $20 \mu M$ LY294002 (LY-20), singly or combined, or $0.1 \%$ dimethylsulfoxide (Con). They were then used for western blot analyses using antibodies against pAKT, $p G S K 3 \beta$, and GAPDH. D: Hs578T cell extracts were collected at 1 or 2 days after treatment with AZD-10, LY-10, LY-20, $10 \mu M$ AZD5363 with $10 \mu M$ LY294002 (AZD+LY10), $10 \mu M$ AZD5363 with $20 \mu M$ LY294002 (AZD+LY-20), or 0.1\% dimethylsulfoxide (Con). They were then used for western blot analyses using antibodies against pAKT and GAPDH.

in reducing cellular growth and pGSK3 $\beta$ level in Hs578T cells (Figure 3A and Figure 2C). We observed that only AZD5363 treatment increased pAKT whereas LY294002, MK-2206, wortmannin, and perifosine alone did not affect the pAKT level (Figure 1C and D, and $3 \mathrm{~A}$ ).

Next, we tested whether AZD5363 induction of pAKT was attenuated by co-treatment with these inhibitors. As shown in Figure 3B-D, only Mk-22206 co-treatment with AZD5363 reduced AZD5363 induction of pAKT. The dosedependent pAKT reduction by MK-2206 was observed at 1 day and sustained for 2 days. However, wortmannin and perifosine treatment for both 1 and 2 days did not affect the pAKT level (Figure 3B-D). LY294002-induced reduction of
pAKT was more effective than that of MK-2206 (Figure 3E).

Collectively, our results indicate that co-treatment of LY294002 or MK-2206 with AZD5363 reduces AZD5363 induction of pAKT. Considering that MK-2206 is now in clinical use, the harmful effects of AZD5363-induced longterm activation of pAKT might be ameliorated by the beneficial effect of its co-treatment with MK-2206.

Our next step was to test whether the co-treatment of LY294002 or MK-2206 with AZD5363 influenced the activation or levels of the signaling proteins that function upstream and downstream of the PI3K/AKT/mTOR pathway $(17,18,25,26)$. We analyzed phosphorylated forms of the major proteins, mTOR, P70S6K, 4EBP1, and 

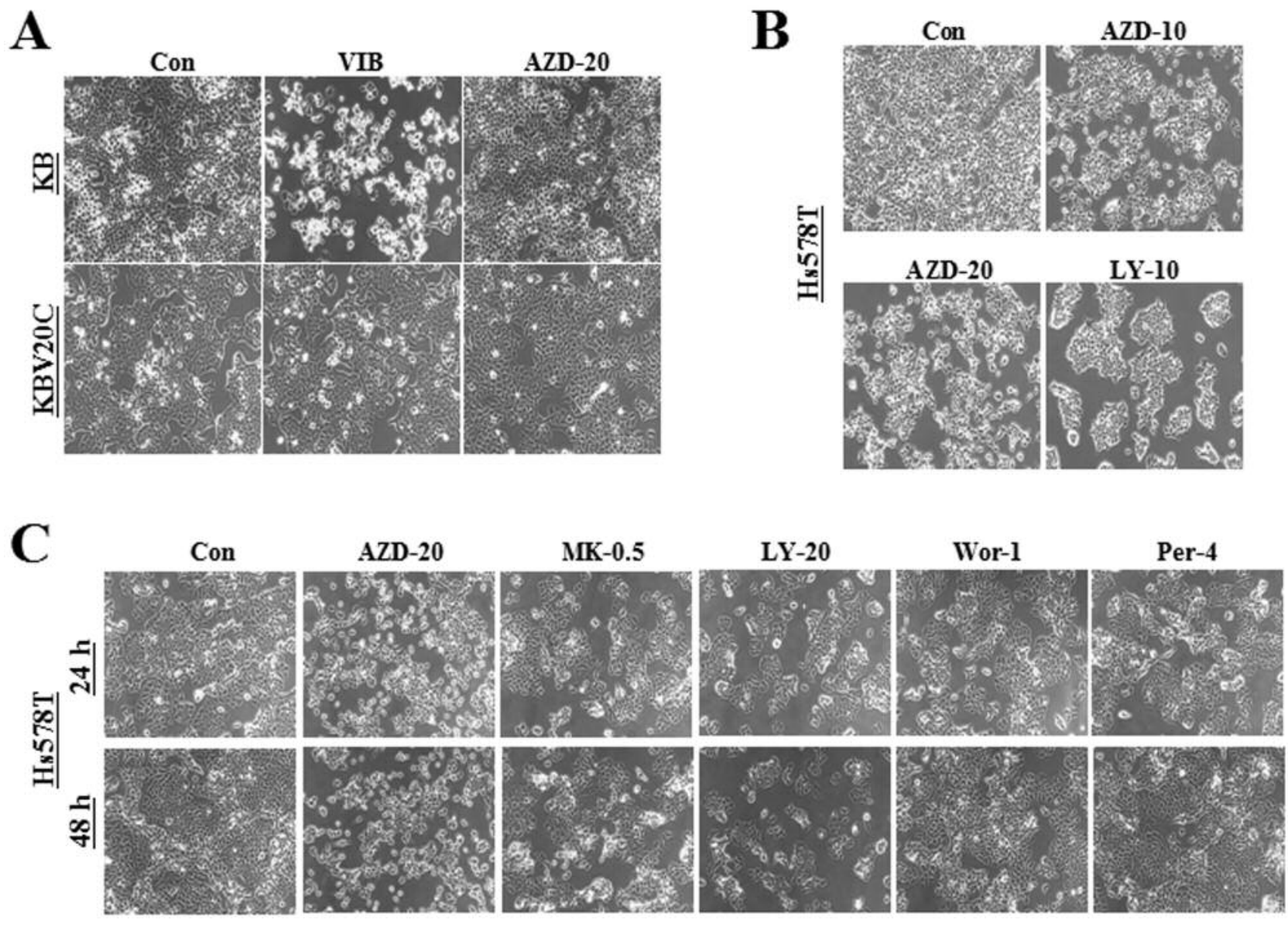

Figure 2. Sensitivity of Hs578T breast cancer cells to various kinase inhibitors in phosphoinositide 3-kinase (PI3K)/AKT/mammalian target of rapamycin ( $m T O R$ ) pathway. A: Sensitive KB and multidrug-resistant KBV20C cells were plated on 6-well plates and treated with $5 \mathrm{nM}$ vinblastine (VIB), $20 \mu M$ AZD5363 (AZD-20), or 0.1\% dimethylsulfoxide (Con). After, 48 h all cells were observed using an inverted microscope with a 10x objective lens. B, C: Hs578T cells were grown on 6-well plates and treated for 24 h with AZD5363 or LY294002 (B) or with agents or inhibitors for 24 or $48 \mathrm{~h}(\mathrm{C})$, then cells were observed using an inverted microscope with a 10x objective lens. AZD-10: 10 $\mu \mathrm{M} \mathrm{AZD5363;} \mathrm{LY-10:10} \mu \mathrm{M}$

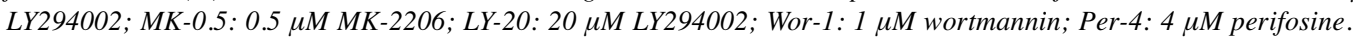

PDK1. Figure 4A indicates that the activation states of all the proteins were similar after single AZD5363 treatment and after co-treatment with LY294002 or MK-2206 with AZD5363. Since the PI3K/AKT/mTOR pathway regulates cellular proliferation and survival $(17,18,25,26)$, we also checked for any alteration in the levels of cell cycle- and proliferation-related proteins ( $\mathrm{pRb}$, cyclin D1, PCNA, and survivin). As displayed in Figure 4A, all protein levels were generally similar after AZD5363 treatment alone and cotreatment with LY294002 or MK-2206. These results suggest that AZD5363 treatment alone was sufficient to reduce the levels of cell cycle- and proliferation-related proteins. We did not investigate the molecules specifically reduced by co-treatments. This suggests that further analysis is required at the molecular level to identify potential cotreatment regimes.
We hypothesized that co-treatment of AZD5363 and LY294002 or MK-2206 increases apoptosis of cancer cells, by reducing pAKT levels. However, we did not observe any increase in C-PARP level under the co-treatment conditions compared with use of AZD5363 alone (Figure 4B and C). Microscopic observations did not reveal any decrease in cellular proliferation for co-treatment with AZD5363 and LY294002 or MK-2206 (Figure 5A and B). This suggests that the reduction of pAKT levels by co-treatment with LY294002 or MK-2206 is too small for growth inhibition to be detected with current assay systems such C-PARP production or microscopic observation with 1- or 2-day duration.

Co-treatment with $m$ TOR inhibitor does not reduce AZD5363 induction of pAKT. Next, we tested whether co-treatment with mTOR inhibitor would reduce AZD5363 induction of pAKT. 


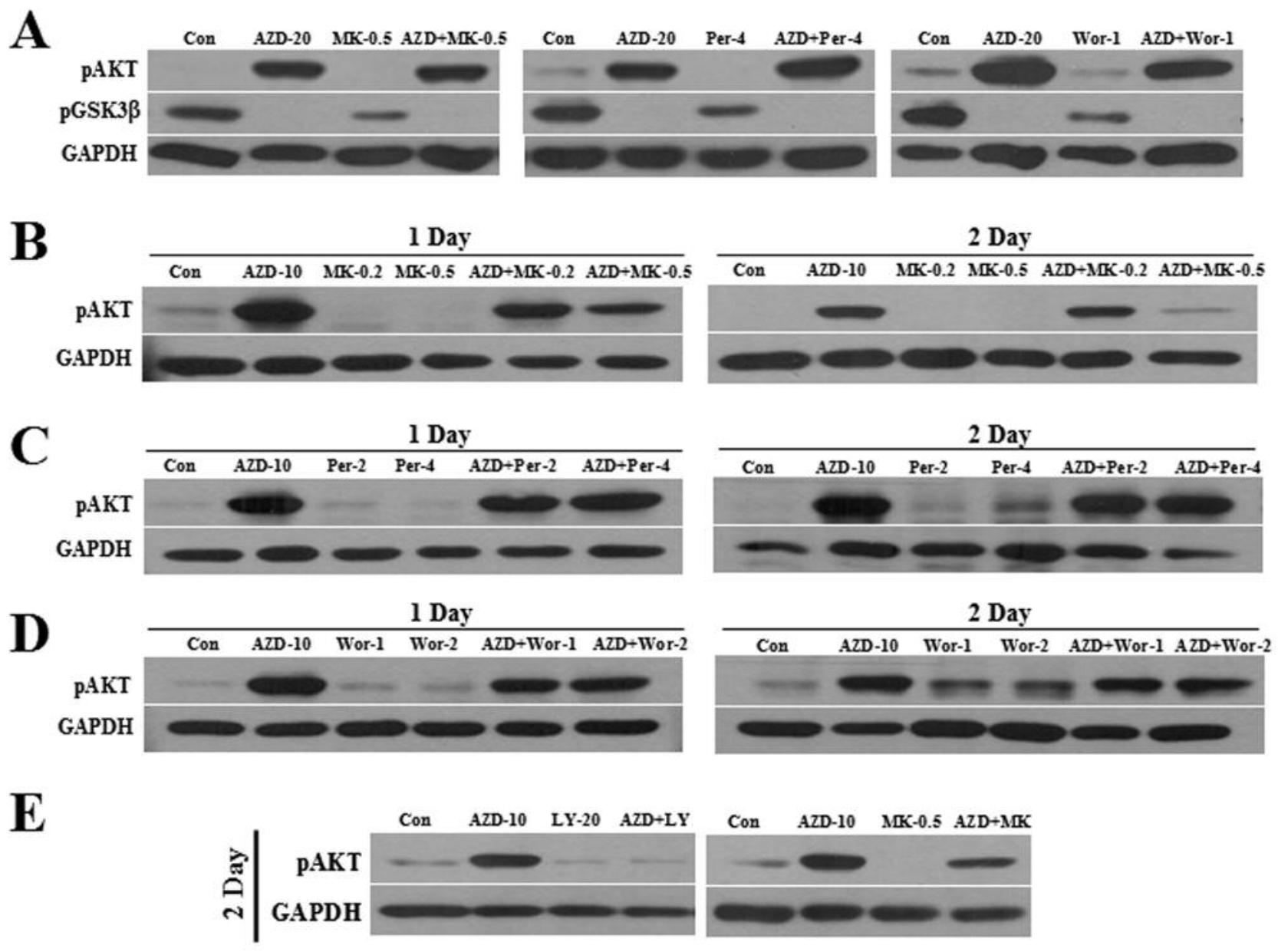

Figure 3. Co-treatment with MK-2206 reduces AZD5363 induction of phosphorylated protein kinase B (pAKT). Hs578T cell extracts were collected at $24 h(A)$, at $24 h$ or $48 h(B-D)$, or at $48 h(E)$ after treatment with agents singly or in combination, then used for western blot analyses. A: Treatment with AZD-20: $20 \mu M$ AZD5363; MK-0.5: $0.5 \mu M$ MK-2206; Per-4: $4 \mu M$ perifosine; Wor-1: 1 $\mu M$ wortmannin; AZD+MK-0.5: $20 \mu M$ AZD5363 with $0.5 \mu M$ MK-2206; AZD+Per-4: $20 \mu M$ AZD5363 with $4 \mu M$ perifosine; AZD+Wor-1: $20 \mu M$ AZD5363 with $1 \mu M$ wortmannin; or $0.1 \%$ dimethylsulfoxide (Con). B-D: Treatment with AZD-10: $10 \mu M$ AZD5363; MK-0.2: $0.2 \mu M$ MK-2206; MK-0.5; Per-2/Per-4: 2/4 $\mu M$ perifosine; Wor-1/Wor-2: 1/2 $\mu M$ wortmannin; AZD+MK-0.2: $10 \mu M$ AZD5363 with $0.2 \mu M$ MK-2206; AZD+MK-0.5; AZD+Per-2/AZD+Per-4: $10 \mu M$ AZD5363 with 2/4 $\mu M$ perifosine; $A Z D+$ Wor-1/AZD+Wor-2: $10 \mu M$ AZD5363 with 1/2 $\mu M$ wortmannin; and 0.1\% dimethylsulfoxide (Con). E: Treated with

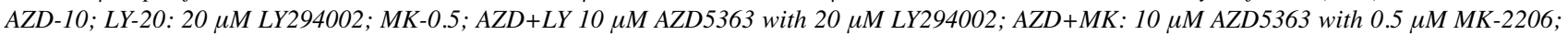
or $0.1 \%$ dimethylsulfoxide (Con). pGSK3 $\beta$ : Phosphorylated glycogen synthase kinase 3 beta, GAPDH: glyceraldehyde 3-phosphate dehydrogenase.

Three inhibitors that are in clinical use, namely rapamycin, everolimus, and temsirolimus (27-29), were selected and investigated to identify their potential for co-treatment with AZD5363. We observed that rapamycin, everolimus, and temsirolimus reduce growth and pGSK $3 \beta$ level in Hs578T cells relative to the control (Figure 5C and Figure 6A), suggesting that mTOR pathway-targeting inhibitors can sensitize Hs578T cells with reduction of AKT pathway. AZD5363 alone increased the pAKT level, while mTOR inhibitors did not influence the pAKT level (Figure 6A). Next, we evaluated whether cotreatment with these inhibitors attenuated pAKT induction by AZD5363. As indicated in Figure 6A, none of the co-treatments with the inhibitors reduced AZD5363 induction of pAKT. In addition, the PI3K/AKT/mTOR-related protein levels were generally similar after AZD5363 treatment alone and cotreatment with rapamycin, everolimus, or temsirolimus (Figure 6B). The C-PARP level did not increase under co-treatment conditions (Figure 6C), and microscopic examinations did not show any decrease in proliferation (Figure 5C).

Collectively, our results indicate that co-treatment of mTOR inhibitors with AZD5363 did not reduce AZD5363 induction of pAKT. This suggests that clinically used MK-2206 proves to be the most potential candidate for co-treatment with AZD5363 to minimize AZD5363 induction of pAKT. 


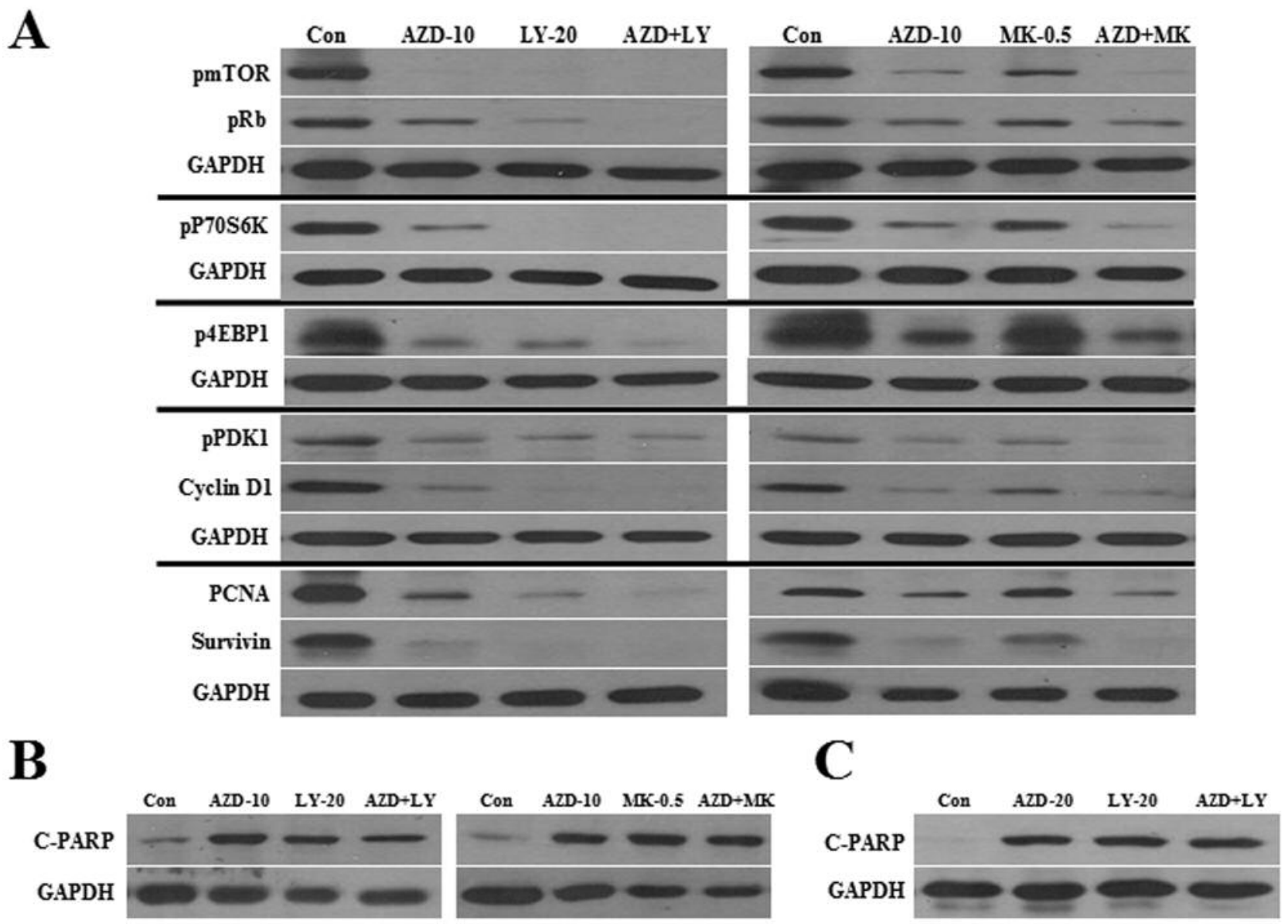

Figure 4. Single treatment with AZD5363 is adequate to reduce the level of growth signaling pathway proteins. A-C: Hs578T cell extracts were collected at $24 \mathrm{~h}$ after treatment with agents singly or in combination then used for western blot analyses using antibodies against phosphorylated mammalian target of rapamycin (pmTOR), phosphorylated retinoblastoma protein ( $p R b)$, phosphorylated ribosomal protein S6 kinase beta-1 (p70S6K), phosphorylated eukaryotic translation initiation factor 4E-binding protein 1 ( $p 4 E B P 1)$, phosphorylated pyruvate dehydrogenase lipoamide kinase isozyme 1 (pPDK1), cyclin D1, proliferating cell nuclear antigen (PCNA), survivin (A), and cleaved poly ADP ribose polymerase (C-PARP) (B, C) with glyceraldehyde 3-phosphate dehydrogenase (GAPDH) as loading control. AZD-10: $10 \mu M$ AZD5363; LY-20: $20 \mu M$ LY294002; MK-0.5: 0.5 $\mu$ M MK-2206; AZD+LY: $10 \mu M$ AZD5363 with $20 \mu M$ LY294002; AZD+MK: $10 \mu M$ AZD5363 with $0.5 \mu M$ MK-2206; or 0.1\% dimethylsulfoxide (Con).

\section{Discussion}

AZD5363 is a novel promising drug that inhibits AKT isoforms (1-4). Interestingly, we found that AZD5363 increases the pAKT level in cancer cells. Of the eight known inhibitors of PI3K/AKT/mTOR pathway: AZD5363, LY294002, MK-2206, wortmannin, perifosine, rapamycin, everolimus, and temsirolimus, only AZD5363 increased the pAKT level. However, all of them reduced the pGSK3 $\beta$ level. Previous studies reported that AKT activation increases cancer cell survival and resistance to apoptosis induced by drugs $(17,18,30)$. We hypothesized that AZD5363 induction of pAKT positively correlates with alternative survival pathways of cancer cells. Therefore, we attempted to identify mechanisms that reduce AZD5363 induction of pAKT in cancer cells in order to enhance the clinical use of AZD5363. We evaluated eight established inhibitors of the PI3K/AKT/mTOR pathway. The proliferation of Hs578T breast cancer cell was reduced by all eight inhibitors with reduction of PI3K/AKT/mTOR pathway. We assumed that growth inhibition induced by $\mathrm{PI} 3 \mathrm{~K} / \mathrm{AKT} / \mathrm{mTOR}$ targeting inhibitors is relatively similar in Hs578T breast cancer cells.

The prominent observation of this study is that AZD5363induced increase in pAKT level was down-regulated by cotreatment with LY294002 or MK-2206. In particular, the role of MK-2206 is crucial, since it has been deemed safe and is clinically used to treat human patients (23). Patients 

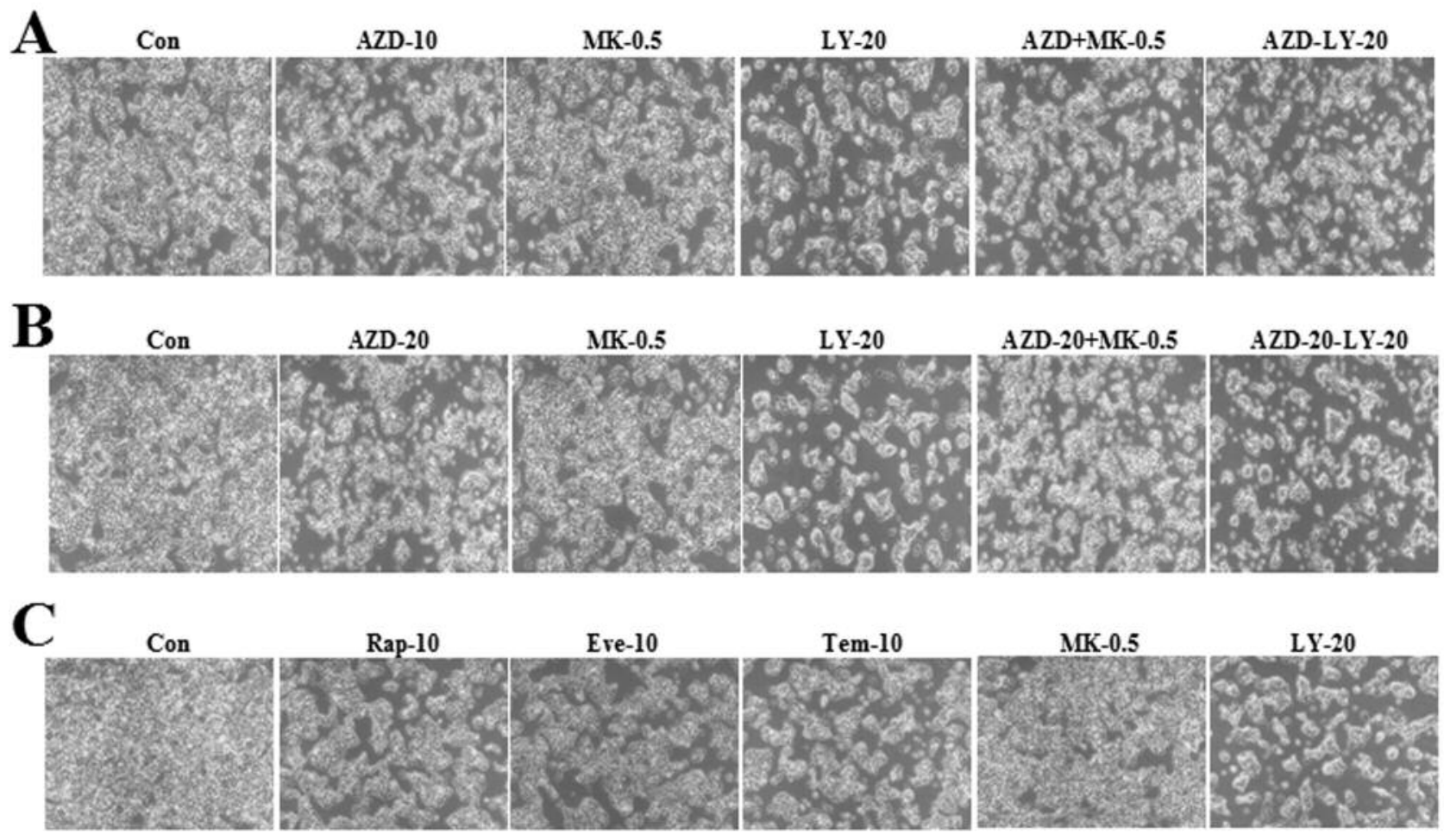

Eve-10
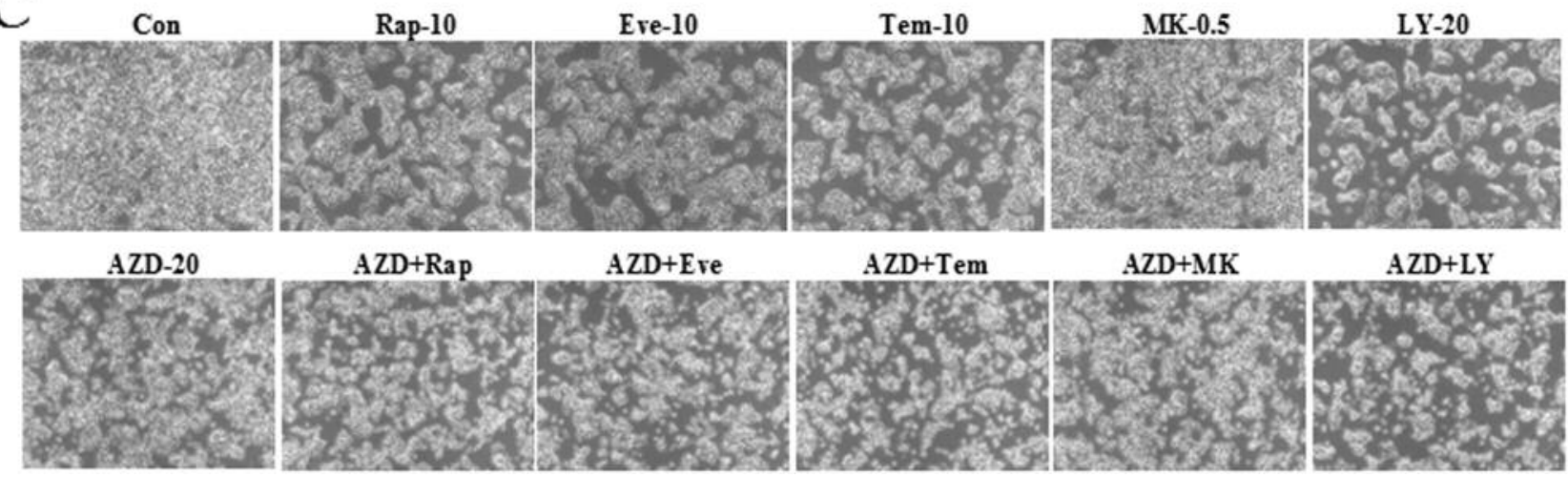

Figure 5. Co-treatment of AZD5363 with phosphoinositide 3-kinase (PI3K)/AKT/mammalian target of rapamycin (mTOR) inhibitors does not reduce growth of AZD5363-treated cells. Hs578T cells were grown on 6-well plates and treated with agents singly or in combination. After 24 h, all cells were observed using an inverted microscope with a 5x objective lens. A and B: AZD-10: $10 \mu M$ AZD5363; MK-0.5: 0.5 $\mu M$ MK-2206; LY-20: $20 \mu M$ LY294002; AZD+MK-0.5: $10 \mu M$ AZD5363 with $0.5 \mu M$ MK-2206; AZD+LY-20: $10 \mu M$ AZD5363 with 20 $\mu M$ LY-294002; AZD-20: $20 \mu M$ AZD5363; AZD-20+MK-0.5: $20 \mu M$ AZD5363 with $0.5 \mu M$ MK-2206; AZD-20+LY-20: $20 \mu M$ AZD5363 with 20 $\mu M$ LY-294002; or 0.1\% dimethylsulfoxide (Con). C: Rap-10: $10 \mu \mathrm{M}$ rapamycin; Eve-10: $10 \mu \mathrm{M}$ everolimus; Tem-10: $10 \mu \mathrm{M}$ temsirolimus; AZD+Rap: $20 \mu \mathrm{M}$ AZD5363 with $10 \mu M$ rapamycin; AZD+Eve: $20 \mu M$ AZD5363 with $10 \mu M$ everolimus; AZD+Tem: $20 \mu M$ AZD5363 with $10 \mu M$ temsirolimus; AZD+MK: $20 \mu M$ AZD5363 with $0.5 \mu M$ MK-2206; AZD+LY: $20 \mu M$ AZD5363 with $20 \mu M$ LY-294002; or 0.1\% dimethylsulfoxide (Con). After 24 h, all cells were observed using an inverted microscope with a $5 \times$ objective lens.

harboring a resistant phenotype and with an increased pAKT level from AZD5363 therapy might be effectively treated with MK-2206 co-treatment. We observed that LY294002 also effectively inhibited AZD5363 induction of pAKT. However, LY294002 is only an experimental inhibitor and not used in the clinical setting. LY294002 and its structural derivatives could potentially be used to study mechanisms of AZD5363 resistance induced by pAKT. Therefore, further studies are warranted to understand the mechanism(s) by which MK-2206 or LY294002 reduce pAKT expression. In future studies, we propose to measure dephosphorylation of AKT by PTEN or phosphorylation of AKT by PI3K, or unidentified kinases. The results of this might be applied for AZD5363-resistant cancer cells, which are the result of increased activation by pAKT.

We classified the eight inhibitors of PI3K/AKT/mTOR pathway based on molecular signatures. AZD5363 influences proteins similarly to LY294002 and mTOR inhibitors, whereas MK-2206 displayed a different pattern. This suggests that MK-2206 exhibits a different mechanism of cellular targeting from that of other PI3K/AKT/mTOR inhibitors. Since the PI3K/AKT/mTOR pathway regulates proliferation and survival signals (17, $18)$, we tested whether expression of cell cycle- and 


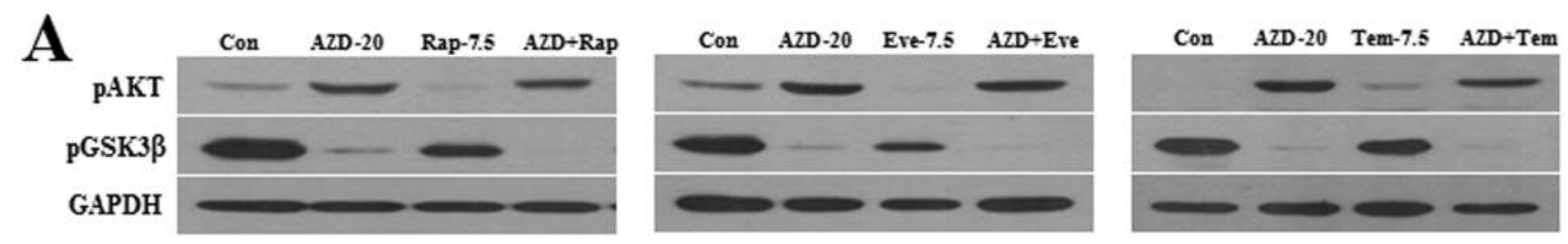

B

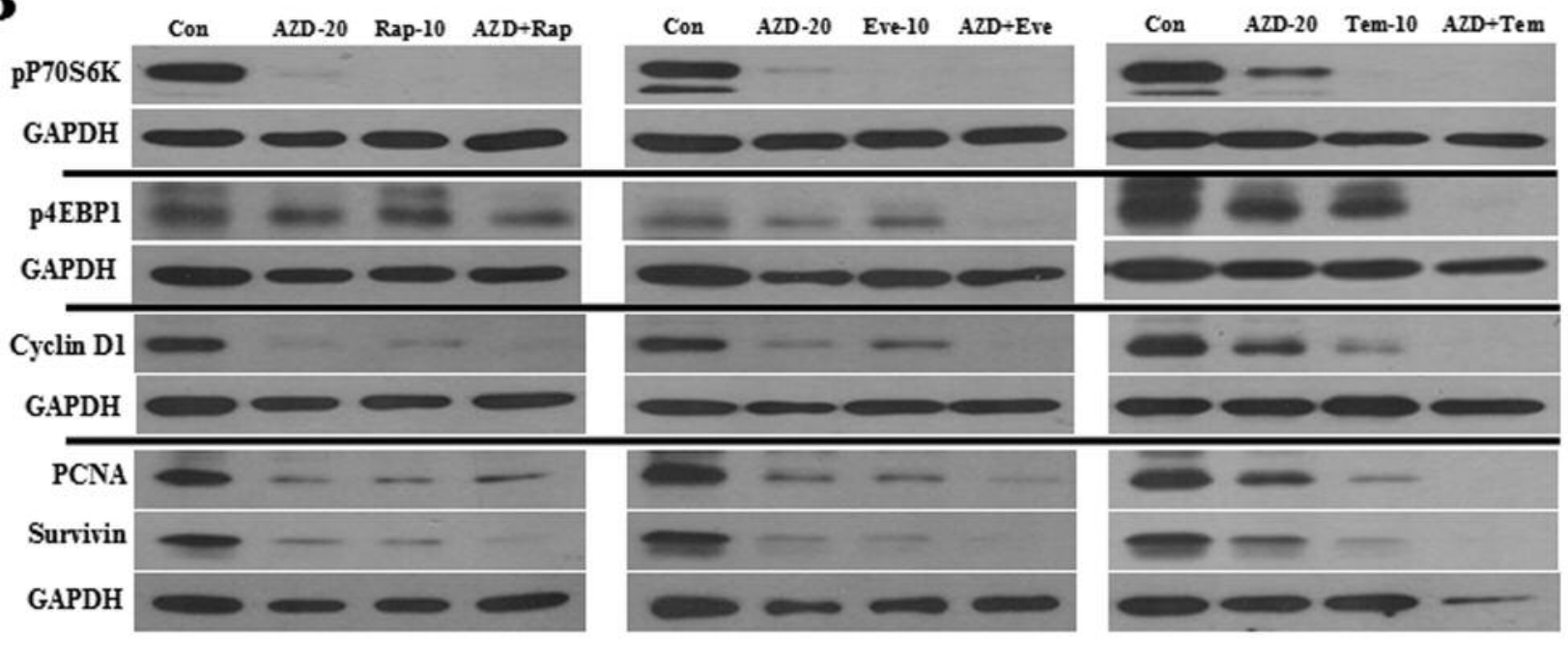

C
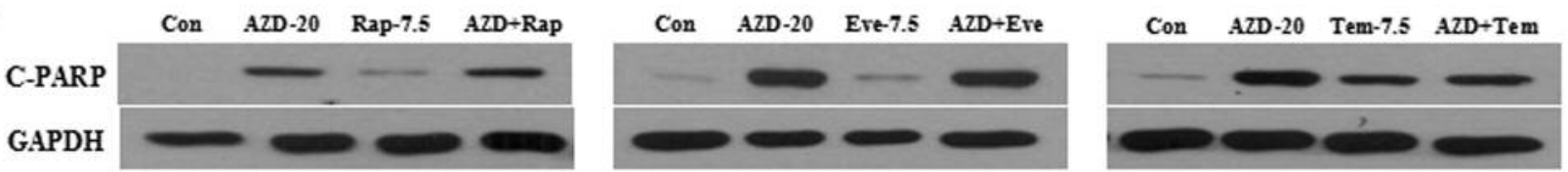

Figure 6. Co-treatment with mammalian target of rapamycin (mTOR) inhibitors does not reduce AZD5363 induction of phosphorylated protein kinase B (pAKT). Hs578T cell extracts were collected at $24 \mathrm{~h}$ after treatment with agents singly or in combination and then used for western blot analyses using antibodies against pAKT and phosphorylated glycogen synthase kinase 3 beta (pGSK3 $\beta)(A)$, phosphorylated ribosomal protein S6 kinase beta-1 ( $p 70 S 6 \mathrm{~K})$, phosphorylated eukaryotic translation initiation factor 4E-binding protein 1 ( $p 4 E B P 1)$, cyclin D1, proliferating cell nuclear antigen (PCNA), and survivin (B) and cleaved poly ADP ribose polymerase (C-PARP) (C), all with GAPDH as loading control. AZD-20: $20 \mu M$ AZD5363; Rap-7.5: $7.5 \mu$ M rapamycin; Eve-7.5: $7.5 \mu$ M everolimus; Tem-7.5: $7.5 \mu M$ temsirolimus; Rap-10: $10 \mu M$ rapamycin; Eve-10: $10 \mu M$ everolimus; Tem-10: $10 \mu M$ temsirolimus; AZD+Rap: $20 \mu M$ AZD5363 with $7.5 \mu M$ or $10 \mu M$ rapamycin; AZD+Eve: $20 \mu M$ AZD5363 with 7.5 $\mu M$ or $10 \mu M$ everolimus; AZD+Tem: $20 \mu M$ AZD5363 with $7.5 \mu M$ or $10 \mu M$ temsirolimus; Con: $0.1 \%$ dimethylsulfoxide.

proliferation-related protein (cyclin D1, pRb, PCNA, and survivin) were altered. The protein levels after single treatment of AZD5363 were comparable to those after cotreatments of LY294002, MK-2206, or mTOR inhibitors. These results suggest that single treatment of AZD5363 was adequate to reduce the levels of growth signaling pathway proteins.

We hypothesized that the reduction in the level of pAKT by LY294002 or MK-2206 would result in sensitization to the growth-inhibitory effects of AZD5363; however, the growth of and apoptosis in cells after single AZD5363 treatment were comparable to that after co-treatments. The effect on pAKT level was minimal, and growth inhibition could not be detected using microscopic observations and analysis of C-PARP production. Further studies are warranted to analyze long-term effects.

In summary, our results contribute to the determination of the potential clinical use of MK-2206 for AZD5363-treated patients with cancer. The present study also enhances our understanding of AZD5363 mechanisms of action. Our findings provide insight into the development of AZD5363based therapies for patients.

\section{Conflicts of Interests}

The Authors declare no conflicts of interests. 


\section{Acknowledgements}

This research was supported by Basic Science Research Program through the National Research Foundation of Korea (NRF) funded by the Ministry of Education (NRF-2014R1A1A2056690).

\section{References}

1 Davies BR, Greenwood H, Dudley P, Crafter C, Yu DH, Zhang J, Li J, Gao B, Ji Q, Maynard J, Ricketts SA, Cross D, Cosulich S, Chresta CC, Page K, Yates J, Lane C, Watson R, Luke R, Ogilvie D and Pass M: Preclinical pharmacology of AZD5363, an inhibitor of AKT: pharmacodynamics, antitumor activity, and correlation of monotherapy activity with genetic background. Mol Cancer Ther 11: 873-887, 2012.

2 Addie M, Ballard P, Buttar D, Crafter C, Currie G, Davies BR, Debreczeni J, Dry H, Dudley P, Greenwood R, Johnson PD, Kettle JG, Lane C, Lamont G, Leach A, Luke RW, Morris J, Ogilvie D, Page K, Pass M, Pearson S and Ruston L: Discovery of 4-amino-N-[(1S)-1-(4-chlorophenyl)-3-hydroxypropyl]-1-(7Hpyrrolo[2,3-d]pyrimidin-4-yl)piperidine-4-carboxamide (AZD5363), an orally bioavailable, potent inhibitor of AKT kinases. J Med Chem 56: 2059-2073, 2013.

3 Maynard J, Ricketts SA, Gendrin C, Dudley P and Davies BR: 2-Deoxy-2-[18F]fluoro-D-glucose positron emission tomography demonstrates target inhibition with the potential to predict antitumour activity following treatment with the AKT inhibitor AZD5363. Mol Imaging Biol 15: 476-485, 2013.

4 Sommer EM, Dry H, Cross D, Guichard S, Davies BR and Alessi DR: Elevated SGK1 predicts resistance of breast cancer cells to AKT inhibitors. Biochem J 452: 499-508, 2013.

5 Ribas R, Pancholi S, Guest SK, Marangoni E, Gao Q, Thuleau A, Simigdala N, Polanska U, Campbell H, Rani A, Liccardi G, Johnston S, Davies BR, Dowsett M and Martin LA: AKT antagonist AZD5363 influences estrogen receptor function in endocrine resistant breast cancer and synergises with fulvestrant (ICI182780) in vivo. Mol Cancer Ther 14: 2035-2048, 2015.

6 Lamoureux F, Thomas C, Crafter C, Kumano M, Zhang F, Davies BR, Gleave ME and Zoubeidi A: Blocked autophagy using lysosomotropic agents sensitizes resistant prostate tumor cells to the novel AKT inhibitor AZD5363. Clin Cancer Res 19: 833-844, 2013.

7 Lamoureux F and Zoubeidi A: Dual inhibition of autophagy and the AKT pathway in prostate cancer. Autophagy 9: 1119-1120, 2013.

8 Meja K, Stengel C, Sellar R, Huszar D, Davies BR, Gale RE, Linch DC and Khwaja A: PIM and AKT kinase inhibitors show synergistic cytotoxicity in acute myeloid leukaemia that is associated with convergence on MTOR and MCL1 pathways. Br J Haematol 167: 69-79, 2014.

9 Deng X, Hu J, Ewton DZ and Friedman E: Mirk/dyrk1B kinase is upregulated following inhibition of mTOR. Carcinogenesis 35: 1968-1976, 2014.

10 Li J, Davies BR, Han S, Zhou M, Bai Y, Zhang J, Xu Y, Tang L, Wang H, Liu YJ, Yin X, Ji Q and Yu DH: The AKT inhibitor AZD5363 is selectively active in PI3KCA mutant gastric cancer, and sensitizes a patient-derived gastric cancer xenograft model with PTEN loss to Taxotere. J Transl Med 11: 241, 2013.

11 Liu L and Dong X: Complex impacts of PI3K/AKT inhibitors to androgen receptor gene expression in prostate cancer cells. PloS One 9: e108780, 2014.
12 Puglisi M, Thavasu P, Stewart A, de Bono JS, O'Brien ME, Popat $\mathrm{S}$, Bhosle $\mathrm{J}$ and Banerji $\mathrm{U}$ : AKT inhibition synergistically enhances growth-inhibitory effects of gefitinib and increases apoptosis in non-small cell lung cancer cell lines. Lung Cancer 85: 141-146, 2014.

13 Thomas C, Lamoureux F, Crafter C, Davies BR, Beraldi E, Fazli L, Kim S, Thaper D, Gleave ME and Zoubeidi A: Synergistic targeting of PI3K/AKT pathway and androgen receptor axis significantly delays castration-resistant prostate cancer progression in vivo. Mol Cancer Ther 12: 2342-2355, 2013.

14 Toren P, Kim S, Cordonnier T, Crafter C, Davies BR, Fazli L, Gleave ME and Zoubeidi A: Combination AZD5363 with Enzalutamide Significantly Delays Enzalutamide-resistant Prostate Cancer in Preclinical Models. Eur Urol 67: 986-990, 2015.

$15 \mathrm{Wu}$ X, Zhang J, Zhen R, Lv J, Zheng L, Su X, Zhu G, Gavine PR, Xu S, Lu S, Hou J, Liu Y, Xu C, Tan Y, Xie L, Yin X, He D, Ji Q, Hou Y and Ge D: Trastuzumab anti-tumor efficacy in patient-derived esophageal squamous cell carcinoma xenograft (PDECX) mouse models. J Transl Med 10: 180, 2012.

16 Crafter C, Vincent JP, Tang E, Dudley P, James NH, Klinowska T and Davies BR: Combining AZD8931, a novel EGFR/HER2/ HER3 signalling inhibitor, with AZD5363 limits AKT inhibitor induced feedback and enhances antitumour efficacy in HER2amplified breast cancer models. Int J Oncol 47: 446-454, 2015.

17 Choi AR, Kim JH and Yoon S: Sensitization of cancer cells through reduction of total AKT and downregulation of salinomycin-induction of pAKT, pGSK3beta, pTSC2, and p4EBP1 by cotreatment with MK-2206. Biomed Res Int 2014: 295760, 2014.

18 Kim JH, Choi AR, Kim YK, Kim HS and Yoon S: Low amount of salinomycin greatly increases AKT activation, but reduces activated p70S6K levels. Int J Mol Sci 14: 17304-17318, 2013.

19 Kim JH, Kim TY, Kim HS, Hong S and Yoon S: Lower salinomycin concentration increases apoptotic detachment in high-density cancer cells. Int J Mol Sci 13: 13169-13182, 2012.

$20 \mathrm{Kim}$ NH, Kim SN, Oh JS, Lee S and Kim YK: Anti-mitotic potential of 7-diethylamino-3(2'-benzoxazolyl)-coumarin in 5fluorouracil-resistant human gastric cancer cell line SNU620/5FU. Biochem Biophys Res Commun 418: 616-621, 2012.

21. Petrich AM, Leshchenko V, Kuo PY, Xia B, Thirukonda VK, Ulahannan N, Gordon S, Fazzari MJ, Ye BH, Sparano JA and Parekh S: AKT inhibitors MK-2206 and nelfinavir overcome mTOR inhibitor resistance in diffuse large B-cell lymphoma. Clin Cancer Res 18: 2534-2544, 2012.

22 Cheng Y, Zhang Y, Zhang L, Ren X, Huber-Keener KJ, Liu X, Zhou L, Liao J, Keihack H, Yan L, Rubin E and Yang JM: MK2206, a novel allosteric inhibitor of AKT, synergizes with gefitinib against malignant glioma via modulating both autophagy and apoptosis. Mol Cancer Ther 11: 154-164, 2012.

23 Yap TA, Yan L, Patnaik A, Fearen I, Olmos D, Papadopoulos K, Baird RD, Delgado L, Taylor A, Lupinacci L, Riisnaes R, Pope LL, Heaton SP, Thomas G, Garrett MD, Sullivan DM, de Bono JS and Tolcher AW: First-in-man clinical trial of the oral panAKT inhibitor MK-2206 in patients with advanced solid tumors. J Clin Oncol 29: 4688-4695, 2011.

$24 \mathrm{Kim}$ JH, Kim TH, Kang HS, Ro J, Kim HS and Yoon S: SP600125, an inhibitor of Jnk pathway, reduces viability of relatively resistant cancer cells to doxorubicin. Biochem Biophys Res Commun 387: 450-455, 2009. 
25 Chen Q, Ganapathy S, Singh KP, Shankar S and Srivastava RK: Resveratrol induces growth arrest and apoptosis through activation of FOXO transcription factors in prostate cancer cells. PloS One 5: e15288, 2010.

26 Knuefermann C, Lu Y, Liu B, Jin W, Liang K, Wu L, Schmidt M, Mills GB, Mendelsohn J and Fan Z: HER2/PI-3K/AKT activation leads to a multidrug resistance in human breast adenocarcinoma cells. Oncogene 22: 3205-3212, 2003.

27 Malaguti P, Vari S, Cognetti F and Fabi A: The Mammalian target of rapamycin inhibitors in breast cancer: current evidence and future directions. Anticancer Res 33: 21-28, 2013.

28 Saiki S, Sasazawa Y, Imamichi Y, Kawajiri S, Fujimaki T, Tanida I, Kobayashi H, Sato F, Sato S, Ishikawa K, Imoto M and Hattori N: Caffeine induces apoptosis by enhancement of autophagy via $\mathrm{PI} 3 \mathrm{~K} / \mathrm{AKT} / \mathrm{mTOR} / \mathrm{p} 70$ S6K inhibition. Autophagy 7: 176-187, 2011.
29 Dienstmann R, Rodon J, Serra V, Tabernero J: Picking the point of inhibition: a comparative review of $\mathrm{PI} 3 \mathrm{~K} / \mathrm{AKT} / \mathrm{mTOR}$ pathway inhibitors. Mol Cancer Ther 13: 1021-1031, 2014.

30 Jokinen E, Laurila N, Koivunen P and Koivunen JP: Combining targeted drugs to overcome and prevent resistance of solid cancers with some stem-like cell features. Oncotarget 5: 92959307, 2014.
Received February 19, 2016

Revised April 10, 2016

Accepted April 11, 2016 